\title{
Saber é prevenir: uma nova abordagem no combate ao câncer de mama
}

\author{
Knowledge is prevention: \\ a novel approach to breast cancer prevention
}

\author{
Daniel Dongiu Kim ${ }^{1}$ \\ Ana Laura Lima Araujo ${ }^{1}$ \\ Andréia I An Tsai ${ }^{1}$ \\ Fabio HenriqueKojima ${ }^{1}$ \\ Jorge Shiro Inamori Takashima ${ }^{1}$ \\ Lauro Fumiyuki OtsukaJunior ${ }^{1}$ \\ Ricardo Augusto Bravo Gamboa ${ }^{1}$ \\ Ricardo Kimura ${ }^{1}$ \\ Ricardo Takashi Sugawara ${ }^{1}$ \\ Elaine Fracasso Tambellini ${ }^{1}$ \\ Cássio Silveira ${ }^{1}$ \\ Rute Loreto Sampaio de Oliveira ${ }^{1}$
}

\footnotetext{
${ }^{1}$ Departamento deM edicina Social, FaculdadedeCiências M édicas da Santa Casa de São Paulo. Rua. Dr. Cesário MottaJr. 61. 01221-000 São Paulo SP.

d.kim@itelefonica.com.br
}

Abstract Data from the Brazilian Ministry of Health shows that the breast cancer is one of the leading causes of women's death, despite the enhancements in treatment and diagnosis of this disease. Successive explanatory advertising campaigns emphasized themethods available for prevention: auto examination, clinical examination by a health care professional and mammography. Regardless of their relevance, only these strategies did not reduced incidence of breast cancer, since they do not impact on the oncogenesis factors. The objective of this article is to develop a health promotion activity through the reinforcement of the previous knowledge and aggregation of new information about breast cancer of a basic health unit. The methodology used was a transversal study of pedagogical intervention in the waiting rooms of a basic health unit. The pedagogical intervention was composed by an interactive questionnaire, lecture and discussion about the topic associated with a qualitative and quantitative evaluation of the activity. The proposed activity had positive results since the participants had qualitative and quantitative improvement in their knowledgeand also a good interactivity with the researchers.

Key words Breast cancer prevention, Strategies in public health
Resumo No Brasil, dados do M inistério da Saúde apontam o câncer de mama como principal causa demorteem mulheres, apesar dos avançosno diagnóstico e tratamento desta enfermidade. Sucessivas campanhas de esclareci mento à população enfatizaram os meios disponíveis para prevenção: autoexame, exame clínico por profissional da saúde e a mamografia. Apesar da sua importância, estas estratégias isoladamente não reduziram a incidência deste agravo à saúde, já quenão atuam nos fatores predisponentes para sua iniciação. 0 objetivo desse estudo foi desenvolver uma atividade de promoção à saúde através do reforço de conhecimentos existentes e agregação de novas informações sobre o câncer de mama no contexto de Unidade Básica de Saúde. Trata-se de um estudo transversal de intervenção pedagógica em salas de espera de U nidade Básica de Saúde. A intervenção pedagógica foi realizada através de questionário interativo, palestra informativa e discussão livre sobre o tópico, associados à avaliação quantitativa e qualitativa desta intervenção. A abordagem proposta foi positiva, uma vez que houve melhora quantitativa equalitativa da compreensão do público sobre os temas abordados, boa interatividade entre os palestrantes e o público, ecriação deagentes multiplicadores de conhecimento.

Palavras-chave Prevenção de câncer de mama, Estratégia em saúde pública 
Introdução

As neoplasias atingem mais de 25 milhões de pessoas em todo o mundo atualmente, sendo uma das doenças recordistas de mortalidade em todos os países. A Organização Mundial da Saúde estima que as neoplasias foram a causa principal de morte de 7,6 milhões de pessoas no ano de 2005 , sendo que $70 \%$ destas mortes ocorreram em países pobres ou em desenvolvimento ${ }^{1}$.

Neste contexto, 0 câncer de mama permanececomo o segundo tipo decâncer mais frequente no mundo eo primeiro entre as mulheres, sendo que as taxas de incidência do câncer de mama dobraram nos últimos trinta anos Com isto, a prevenção ao câncer tornou-se um dos principais objetivos dos programas de saúde públicos, devido a sua crescente incidência e a alta mortalidade associada ${ }^{2,3}$.

Segundo estimativas da Agência Internacional para Pesquisa sobre Câncer (IARC), a prevenção de exposição a agentes reconhecidamente carcinogênicos diminuiria a incidência geral de câncer no mundo em $40 \%$. Outras medidas de saúde pública, como mudança de hábitos e vícios (tabagismo, etilismo, sedentarismo, dieta), imunização contra determinadas doenças (he patite $B$, vírus HPV, entre outros), controle de riscos relacionados ao local de trabalho, redução da exposição solar, entre outros, teriam a capacidade de diminuir, parar e até reverter o processo neoplásico ${ }^{4}$.

No país, dez mil mulheres morrem todos os anos em virtude do diagnóstico tardio, principal determinante de intratabilidade do tumor. Este fato ocorre principalmente porque, em $60 \%$ dos casos, a doença é detectada em estágio avançado. É conhecido que o momento do diagnóstico do câncer tem influência direta em sua evolução e prognóstico, sendo a precocidade do diagnóstico diretamente proporcional às chances de cura ${ }^{5-7}$.

Objetivos

Geral

Desenvolvimento de atividade de promoção e prevenção do câncer de mama na população usuária do Centro de Saúde Escola Barra Funda (CSEBF).

\section{Específicos}

Realizar estudo transversal de intervenção pedagógica através de dinâmica em grupo (sala de espera) com orientações sobre prevenção ao câncer de mama. Informar, esclarecer e orientar a população usuária do CSEBF sobre as formas deprevenção primária edetecção precoce do câncer de mama. Criar uma rede de multiplicação de informação sobre prevenção do câncer de mama na população usuária do CSEBF.

\section{M ateriais e métodos}

O estudo foi realizado no Centro de Saúde Escola da Barra Funda, vinculado à Faculdade deCiências M édicas da Santa Casa de São Paulo. A atividade deorientação, esclarecimento einformação sobre o câncer de mama foi realizada através de dinâmica de grupo nas salas de espera, com os pacientes que aguardavam 0 atendimento, no período da manhã e tarde.

A sala de espera foi conduzida pelos pesquisadores segundo a seguinte estrutura:

- Apresentação dos pesquisadores, da temática, estruturação da atividade e início do registro sonoro, fotográfico, filmográfico eanotações dos relatos durante a atividade;

- Coleta do primeiro nome, sexo e idade dos participantes;

- Realização de seis perguntas interativas aos participantes. As perguntas consistiram em questões com três alternativas cada, apresentadas aos ouvintes em linguagem simplificada. As perguntas foram feitas oralmente e as respostas foram mostradas em flipchart através de iconografia (personagem temático criado pelos pesquisadores), de acordo com o que se segue:

Pergunta 1: Você pode vir a ter câncer de mama algum dia em sua vida?

Pergunta 2: 0 câncer de mama tem cura?

Pergunta 3: Qual o melhor exame para se descobrir 0 câncer de mama quando ele ainda está pequeno?

Pergunta 4: 0 que é mamografia?

Pergunta 5: Com que idade deve ser feita a mamografia?

Pergunta 6: Qual o melhor método para prevenir 0 câncer de mama? 
Os participantes responderam as perguntas levantando cartões coloridos e com formas geométricas diferentes, cada qual correspondendo a uma alternativa, de forma a não excluir pessoas com dificuldade de leitura ou compreensão. Todas as respostas foram registradas.

. Intervenção pedagógica em linguagem simplificada através da exposi ção da magnitudee do impacto do câncer de mama na população brasileira, explicação sobre os principais aspectos da oncogênese e formas de prevenção primária e história natural do câncer e os métodos de diagnóstico precoce;

- Demonstração in loco do autoexame de mamas;

. Interven ção pedagógica em linguagem simplificada sobrea periodicidade recomendada pelo M inistério da Saúde para a realização do autoexame das mamas, exame clínico das mamas por profissional de saúde e mamografia.

- Realização das mesmas seis perguntas interativas realizadas no início da atividade aos participantes e registro das respostas;

- Esclarecimentos individuais por parte dos pesquisadores para eventuais dúvidas a respeito do assunto e consultoria médica para aqueles que quisessem iniciar acompanhamento.

Os resultados obtidos foram analisados de forma quantitativa (análise das respostas às perguntas propostas no início e no fim da atividade) e qualitativa (análise e classificação dos comentários dos participantes).

\section{Resultados}

\section{Análisequantitativa}

Foram realizadas seis dinâmicas de grupo, utilizando-se a sala de espera da saúde da muIher, saúde do adulto e saúde da criança nos dias $15,16,21$, 22 e 23 de março de 2007. Os grupos que participaram das atividades compunham-se dos pacientes que aguardavam 0 atendimento e outras pessoas dentro do CSEBF que se interessassem pela atividade, totalizando 72 pessoas. 0 tempo médio gasto em cada atividade foi de 57,5 minutos, a média da idade dos participantes foi de 38,09 anos, com desvio padrão de 16,68 anos, e houve participação predominantemente de mulheres ( $92 \%$ dos participantes).
Avaliação das respostas ao questionário

A análise quantitativa dos dados obtidos através do questionário interativo realizado antes edepois da interven ção pedagógica foi realizada através de análise de distribuição percentual. Estemodelo considera a distribuição percentual das respostas em cadaalternativa, permitindo a análisede dados provenientes de amostras não pareadas.

Pergunta 1: Houve melhora na performance, com aumento de $42,12 \%$ de respostas corretas.

Pergunta 2: Houve piora na performance, com diminuição de $10,88 \%$ de respostas corretas.

Pergunta 3: Houve melhora na performance, com aumento de $22,24 \%$ de respostas corretas.

Pergunta 4: Houve melhora discreta na performance, com aumento de $1,6 \%$ de respostas corretas.

Pergunta 5: Houve discreta piora na performance, com diminuição de 3,03\% das respostas corretas.

Pergunta 6: A performance neste quesito se manteve praticamente estável.

\section{Análise qualitativa}

A análise qualitativa baseou-se no modelo de estudo avaliativo, no qual se classifica em diferentes grupos o registro do discurso dos participantes das salas de espera. Os diversos trechos do discurso foram classificados em quatro categorias principais: dúvidas de cunho técnico, depoimentos e experiências pessoais, mitos ecrenças e assimilação de informação.

\section{Discussão}

A proposta de criação da estratégia de intervenção pedagógica através da dinâmica em grupo foi bem-sucedida, uma vez que conseguiu se desenvolver uma metodologia adequada. 0 modelo desenvolvido permitiu um livre fluxo de idéias entre os pesquisadores e os usuários do CSEBF, possibilitando a transmissão dos conhecimentos a respeito da prevenção do câncer de mama.

0 uso da sala de espera foi fruto de observações feitas por profissionais do Centro de Saúde Escola Barra Funda, que identificaram a sala de espera como um local privilegiado para se discutir as dúvidas e o conhecimento prévio dos usuá- 
rios, uma vez queantes deserem convocados para as consultas os pacientes permaneciam reunidos eociosos na antessal a de cada setor médico. Além disso, éum espaço quepossibilita aos participantes trocas de experiências, construção de conhecimentos e oferece a oportunidade para as pessoas lidarem com seus problemas e dúvidas, ${ }^{8,9}$.

A metodologia empregada foi eficaz para informar, esclarecer e orientar a população usuária do CSEBF a respeito da preven ção e detecção precoce do câncer de mama, fato este comprovado pelos dados quantitativos e qualitativos. A avaliação quantitativa do questionário interativo, realizado antes e após a intervenção pedagógica, demonstrou quehouve assimilação do conteúdo exposto. A avaliação qualitativa demonstrou a participação ativa dos usuários através da exposição de opiniões, argumentos, depoimentos, experiências pessoais e dúvidas.

Acredita-se que as informações difundidas serão multiplicadas para a comunidade da região, uma vez quehouveinteressee esclarecimento de dúvidas dos participantes. Além disso, os participantes foram estimulados e informados a respeito da importância da multiplicação do conhecimento sobre prevenção primária e secundária do câncer de mama.

Devido à natureza da atividade (as pessoas tinham liberdade para entrar ou sair da sala de espera durante a apresentação), a amostragem não foi pareada, ou seja, o grupo de pessoas que iniciou a atividade não necessariamente foi 0 mesmo que a terminou, levando a impossibilidade de análise estatística mais profunda sobre as respostas ao questionário.

A população usuária do CSEBF é majoritariamentedo sexo feminino. Durante a atividadede sala de espera com o grupo do climatério, observou-se adequado grau de informação e boa cobertura do nível secundário de atendimento.

As perguntas do questionário interativo tinham objetivos específicos, de acordo com a Tabela 1. Consideramos que a mídia é um fator de disseminação deinformações, porém seu impacto sobre o conhecimento sobre câncer de mama é mediano, uma vez que a maior parte das campa-

Tabela 1. O bjetivos e comentário sobre os resultados do questionário interativo.

\begin{tabular}{|c|c|c|}
\hline Questão & Objetivos & Resultados \\
\hline $\begin{array}{l}\text { Você pode ter câncer de } \\
\text { mama algum dia na vida? }\end{array}$ & $\begin{array}{l}\text { Avaliar o grau de conhecimento sobre a } \\
\text { vulnerabilidade ao câncer de mama, ou } \\
\text { seja, a susceptibilidade da população a } \\
\text { este evento. }\end{array}$ & $\begin{array}{l}\text { Existe pouca informação sobre a } \\
\text { natureza aleatória das alterações } \\
\text { genéticas que dão origem a } \\
\text { neoplasias. }\end{array}$ \\
\hline O câncer de mama tem cura? & $\begin{array}{l}\text { Avaliar o grau de conhecimento sobre a } \\
\text { gravidade do câncer de mama, ou seja, } \\
\text { avaliação da letalidade e das sequelas } \\
\text { causadas pela doença. }\end{array}$ & $\begin{array}{l}\text { O câncer de mama já não é } \\
\text { mais visto como doença de } \\
\text { inexorável prognóstico sombrio. }\end{array}$ \\
\hline $\begin{array}{l}\text { Qual é o melhor método } \\
\text { para descobrir o câncer de } \\
\text { mama quando ele ainda é } \\
\text { bem pequeno? }\end{array}$ & $\begin{array}{l}\text { Avaliar o grau de conhecimento sobre } \\
\text { métodos de diagnóstico precoce do } \\
\text { câncer de mama e sua aplicabilidade }{ }^{10} \text {. }\end{array}$ & $\begin{array}{l}\text { A maior parte da população } \\
\text { conhece os métodos de } \\
\text { diagnóstico precoce, porém } \\
\text { desconhece qual é o mais } \\
\text { efetivo. }\end{array}$ \\
\hline O que é mamografia? & $\begin{array}{l}\text { Avaliar o grau de conhecimento sobre a } \\
\text { mamografia e indiretamente indicar se } \\
\text { o participante já realizou o exame } \\
\text { alguma vez. }\end{array}$ & $\begin{array}{l}\text { A mamografia é um método } \\
\text { diagnóstico bem conhecido pela } \\
\text { população. }\end{array}$ \\
\hline $\begin{array}{l}\text { Qual é a melhor idade para } \\
\text { fazer a mamografia? }\end{array}$ & $\begin{array}{l}\text { Avaliar se os participantes têm } \\
\text { informações não só sobre o método } \\
\text { como também a metodologia } \\
\text { empregada no exame. }\end{array}$ & $\begin{array}{l}\text { A população não tem } \\
\text { informações sobre a } \\
\text { metodologia do exame, } \\
\text { prevalecendo a máxima "quanto } \\
\text { antes melhor". }\end{array}$ \\
\hline $\begin{array}{l}\text { Qual é o melhor método } \\
\text { para se prevenir o câncer de } \\
\text { mama? }\end{array}$ & $\begin{array}{l}\text { Avaliar a transcendência do câncer de } \\
\text { mama, ou seja, a relevância social e o } \\
\text { reconhecimento social do impacto } \\
\text { desta doença. }\end{array}$ & $\begin{array}{l}\text { É importante no imaginário } \\
\text { popular a associação entre } \\
\text { estados de humor e doenças } \\
\text { crônicas como o câncer. }\end{array}$ \\
\hline
\end{tabular}


nhas enfatiza os métodos de diagnóstico precoce e não aborda outros quesitos importantes como a metodologia correta para o sucesso dos métodos de diagnóstico precoce.

\section{Colaboradores}

Araujo ALL trabalhou na concepção e pesquisa; AIA Tsai trabalhou na concepção, pesquisa e redação final; FH Kojima, JSI Takashima, LF Otsuka Junior, RAB Gamboa trabalharam na concepção, pesquisa e trabalho de campo; R Kimura participou da concepção, pesquisa, trabalho de campo e redação final; RT Sugawara participou da concepção, pesquisa e trabal ho de campo; RLS Oliveira eC Silveira trabal haram na metodologia; EF Tambellini participou da pesquisa de campo.

\section{Agradecimentos}

Aos funcionários, estagiários, voluntários e pacientes do Centro de Saúde Escola Barra Funda, pela inestimável colaboração. Ao Exmo. Dr. Luis Carlos Pazero, coordenador da Área Técnica da Saúde da Mulher da Prefeitura do M unicípio de São Paulo e à Coordenação de Epidemiologia e Informação da Secretaria M unicipal de Saúde da Prefeitura de São Paulo. Aos professores, mestres e amigos do Centro de Estudos Augusto Le opoldo Ayrosa Galvão do Departamento de Me dicina Social da Faculdade de Ciências M édicas da Santa Casa de São Paulo.

\section{Referências}

1. World Health Organization. Fact Sheet $N^{\circ} 297$. Genebra: WHO/CHP; 2006.

2. Instituto Nacional do Câncer. Estimativa 2006: Incidência de Câncer no Brasil. Rio de Janeiro: INCA; 2005.

3. Instituto Nacional do Câncer. Consenso para o controle do câncer de mama. Rio de Janeiro: INCA/ CONPREV; 2004.

4. International Agency for Research on Cancer. Handbooks of Cancer Prevention. Lion: WH O/IARC; 2000.

5. Piato S, Piato JRM. Doenças da mama. $1^{\text {a }}$ ed. Rio de Janeiro: Revinter; 2006.

6. Instituto Nacional do Câncer. Atlas de mortalidade por câncer no Brasil 1979-1999. Rio de Janeiro: INCA; 2002.

7. Piato S. Tratado de Ginecologia. 2a ed. São Paulo: Artes M édicas; 2002.

8. Centro de Saúde Escola Barra Funda. Projeto de Salas de Espera. São Paulo: CSEBF; 2002.

9. Cruz RM F, Tambellini EF, Carneiro Junior N, Almeida LMM, Ferro AS, Cruz AL. Sala de espera como ferramenta gerencial e espaço de comunicação: uma experiência do Centro de Saúde Escola Barra Funda Dr. Alexandre Vranjac. In: Anais do $11^{\circ}$ Congresso M undial de Saúde Pública; 2006; Rio de Janeiro.

10. American College of Radiology. BI-RADS Mammography. $4^{\text {th }}$ ed. Reston: ACR; 2003. 